Background and aims Paediatric stroke, although uncommon when compared to adult stroke, has a high mortality and morbidity rate. Between 2 and 13 children per 100,000 per year are likely to have a stroke, with 5\% - 10\% resulting in death and more than 50\% developing neurological and cognitive defects. The aim was to review the current literature and discuss risk factors, aetiology, presentation and management of paediatric stroke.

Methods Literature review.

Results Paediatric stroke is more common amongst boys and is classified as arterial ischaemic or haemorrhagic, depending on the underlying causes. However no type of paediatric stroke is predominant over the other. There is a broad spectrum of risk factors associated with paediatric stroke and the underlying cause often involves multiple factors such as arteriopathies, maternal infections and haematological disorders. The common risk factors of hypertension or diabetes associated with adult stroke play a very minor role in paediatric stroke development. Paediatric stroke is often misdiagnosed or diagnosed at a very late stage due to the non-specific clinical presentation which depends on factors such as age and type of stroke. The medical or surgical management of paediatric stroke depends on the type, aetiology, timing and the extent of the stroke.

Conclusion Paediatric Stroke is a challenging condition in which few distinct guidelines of the most appropriate treatment exist. Further research and studies should be carried out since much of the knowledge and guidelines are currently based on adult stroke.

\section{PO-0861 THE QUALITY OF GENERAL MOVEMENTS DURING THE NEONATAL PERIOD IN MODERATE AND LATE PRETERM INFANTS}

${ }^{1}$ A Scheuchenegger, ${ }^{1} \mathrm{~J}$ Pansy, ${ }^{2} \mathrm{~F}$ Dobaja, ${ }^{3} \mathrm{C}$ Einspieler, ${ }^{1} \mathrm{~B}$ Resch. ${ }^{1}$ Department of Paediatrics Medical University of Graz Austria, Division of Neonatology, Graz, Austria; ${ }^{2}$ Department of Paediatrics Medical University of Graz Austria, Division of Gereneral Paediatrics, Graz, Austria; ${ }^{3}$ Department of Physiology Medical University of Graz Austria, Research Unit iDN - Interdisciplinary Developmental Neuroscience, Graz, Austria

\subsection{6/archdischild-2014-307384.1486}

Objective The assessment of general movements (GMs) is a widely used technique to evaluate neurological (dys)function and to predict neurodevelopmental outcome in infants. De Vries and Bos (Early Hum Dev 2008, 2010) demonstrated that abnormal GMs are often seen in early recordings in extremely low birth weight (BW) and preterm ( $<32$ weeks) infants. Aim of our study was to assess whether this finding could be replicated for moderate and late preterm infants (32/0-36/6 weeks' gestation).

Methods We assessed GMs during the first 2 weeks (Median = 7 days; 5-8 days) of 50 moderate and late preterm infants (31 males). GM quality (global and detailed scoring) was analysed off line and related to neonatal morbidity (mainly IRDS) and other clinical factors (birth weight, need for oxygen and intensive care).

Results Mean gestational age (GA) of the infants was 35 weeks' gestation ( $\mathrm{SD}=9$ days); mean $\mathrm{BW}$ was 2207 grams $(\mathrm{SD}=$ 400). Abnormal GMs were observed in 23 infants: 19 poor repertoire, 2 infants cramped synchronised, and 2 chaotic. Yet another 10 infants were scored as normal but their detailed GM score revealed a reduced motor optimality. GM abnormalities were not related to perinatal factors, such as GA, birth weight or neonatal morbidity.
Conclusion Almost every second infant had abnormal GMs during the first 2 weeks of life. Whether such an early and single GM assessment will be related to the neurodevelopmental outcome has still to remain open, as the individuals of our study group did not yet reach the age of an outcome assessment.

\section{PO-0862 WITHDRAWN}

\section{PO-0863 LONGITUDINAL CHANGES OF CORTICAL THICKNESS FOLLOWING PREMATURE BIRTH}

${ }^{1} \mathrm{~L}$ Vasung, ${ }^{1} \mathrm{~L}$ Gui, ${ }^{1} \mathrm{C}$ Borradori Tolsa, ${ }^{2} \mathrm{~F}$ Lazeyras, ${ }^{1} \mathrm{P}$ Huppi. ${ }^{1}$ Division of Development and Growth, Department of Pediatrics, HUG, Geneva, Switzerland; 'Department of Radiology, HUG, Geneva, Switzerland

\subsection{6/archdischild-2014-307384.1487}

During the early postnatal period the cerebral cortex undergoes substantial reorganisation. Early changes in environmental factors (e.g. premature birth, socio-economic status) affect the reorganisation of the cerebral cortex.

In order to answer the question of how premature birth affects the cortex, we have analysed T1 MR images $(n=14)$ of prematurely born children (26-35 GW) at term equivalent age. Furthermore, in order to identify the factors affecting the maturation of the cerebral cortex at school age we have analysed T1 MR images of prematurely born children at school age $(n=42$, $6.62 \pm 0.48$ years). While the segmentation of cerebral tissue in school age children was performed using the automatic method (CIVET), we have developed a new morphology-driven automatic segmentation method for the segmentation of cerebral tissue at term equivalent age. The grey and white matter surface meshes were extracted and regional volumes of the cortex and cortical thickness were estimated. Cortical metrics were calculated using the advanced MR image processing tools developed at MNI.

Mean cortical thickness, from term equivalent age to school age, showed a two-fold increase in prematurely born children. Regional variations of cortical thickness in prematurely born children at term equivalent age and school age indicated that the limbic cortex is the first to thicken while the frontal cortex lags behind. Parents' socio-economic status showed positive correlation with mean cortical thickness at school age.

In conclusion, this is the first reported analysis of longitudinal changes of cortical thickness from term equivalent age to school age in prematurely born children.

\section{Neurology and Developmental Paediatrics}

\section{PO-0863a PUBERTY PERIOD AND EPILEPSY ONSET}

${ }^{1} \mathrm{G}$ Odintsova, ${ }^{1} \mathrm{~N}$ Koroleva, ${ }^{2} \mathrm{~L}$ Saykova. 'Neurology, Institute of Human Brain of Ras, Saint Petersburg, Russia; ${ }^{2}$ Neurology, North-Western State Medical University N.a. Mechnicov, Saint Petersburg, Russia

\subsection{6/archdischild-2014-307384.1488}

Purpose Study connection between epilepsy onset and puberty period at female patients.

Methods work was the part of the antiepileptic drugs reproductive side effects study. Epilepsy onset were studied at 155 female patients older 16 y. Patients were divided into 3 groups 